\title{
Tropical algebraic geometry
}

\author{
Shinsuke ODAGIRI
}

(Received September 19, 2008; Revised February 2, 2009)

\begin{abstract}
The construction of a tropical hypersurface is given by modeling the classical construction of a complex hypersurface. A tropical meromorphic function of finite type is shown to be a tropical rational function. One also has tropical nullstellensatz.

Key words: tropical hypersurface, tropical geometry, tropical semiring, max-plus algebra.
\end{abstract}

\section{Introduction}

Tropical geometry is a new geometry which occur in many fields of mathematics, such as enumerative algebraic geometry, combinatorics, and so on. A tropical hypersurface can be seen as a "limit" of amoebas ([8], [9], [14]), or equivalently, a non-Archimedean amoeba ([5], [9]). It is well-known (see, for instance, [1], [4], [6], [12]) that it also equals the corner locus of a convex piecewise linear function $f: \mathbb{R}^{n} \rightarrow \mathbb{R}$ defined by

$$
f\left(x_{1}, \ldots, x_{n}\right)=\max _{\left(i_{1}, \ldots, i_{n}\right) \in \mathcal{I}}\left(a_{i_{1} \ldots i_{n}}+i_{1} x_{1}+\cdots+i_{n} x_{n}\right)
$$

for some finite subset $\mathcal{I} \subset \mathbb{Z}_{\geq 0}^{n}$ and $a_{i_{1}, \ldots, i_{n}} \in \mathbb{R}$.

Let us denote the operators max and + by "tropical operators" $\oplus$ and $\otimes$ respectively. Then $f$ turns out to be a polynomial function of "a tropical polynomial" denoted by tropical operators as

$$
f\left(x_{1}, \ldots, x_{n}\right)=\sum_{\left(i_{1} \ldots i_{n}\right) \in \mathcal{I}} a_{i_{1} \ldots i_{n}} x_{1}^{i_{1}} \otimes \cdots \otimes x_{n}^{i_{n}} .
$$

Thus a tropical hypersurface is the corner locus of a polynomial function. Let us formulate the above polynomial function more rigorously and study it under the help of semiring theory and complex geometry.

In the first half of this paper, we treat the algebraic structure of a tropical semiring. We define tropical polynomials, tropical polynomial functions, 
tropical rational functions, and tropical meromorphic functions based on semiring theory.

In contrast, the latter half, starting from Section 7 , is devoted to the geometric side. We first define the mixed loci of a meromorphic function. It turns out that the mixed loci of a nonmonomial polynomial equals a traditional tropical hypersurface. Then we expand the definition of a weight to each affine linear subset of maximal dimension ("a facet") of a mixed loci and explore the properties. As a consequence, we have two theorems that resemble those of complex geometry:

- Theorem 9.1 A tropical meromorphic function of finite type is a tropical rational function.

- Theorem 10.1 Tropical Hilbert's nullstellensatz holds.

The latter is already shown more generally in [15]. However, our statement is simpler and the proof is more straightforward from the properties of a weight.

\section{Tropical semirings and tropical polynomials}

Throughout this paper, a semiring (resp. semifield) means a commutative semiring (resp. semifield) with an identity and a zero.

Definition 2.1 Let $\mathbb{T}$ denote the set $\mathbb{R} \cup\{-\infty\}$. We define $\oplus$ and $\otimes$ to be the operators called "tropical addition" and "tropical multiplication" on $\mathbb{T}$ by the followings:

- $a \oplus b:=\max (a, b)$,

- $a \otimes b:=a+b$,

where

- $a \oplus-\infty=-\infty \oplus a=a$,

- $a \otimes-\infty=-\infty \otimes a=-\infty$.

Under these operations, $(\mathbb{T}, \oplus, \otimes)$ satisfies all the properties of a field except for the additive inverse (if $a \neq-\infty$, then the additive inverse " $-a$ " does not exist). Note that $-\infty$ is the zero and 0 is the identity. Thus $(\mathbb{T}, \oplus, \otimes)$ is a semifield. We call this semifield, according to tradition, the tropical semiring or the max-plus algebra. 
Remark The zero $-\infty$ is an absorbing zero, i.e., $a \otimes-\infty=-\infty$. We write the set of all nonzero elements $\mathbb{T} \backslash\{-\infty\}$ as $\mathbb{T}^{\times}$. Since $\mathbb{T}$ is a semifield, every element of $\mathbb{T}^{\times}$has an inverse element $a^{-1}$.

Note that $\mathbb{T}^{n}$ is a semiring by the componentwise tropical addition and multiplication. Also it has a topology induced from the Euclidean topology on $\mathbb{R}_{\geq 0}^{n}$ by the bijective map

$$
\operatorname{Exp}: \mathbb{T}^{n} \ni a=\left(a_{1}, \ldots, a_{n}\right) \mapsto \operatorname{Exp}(a):=\left(e^{a_{1}}, \ldots, e^{a_{n}}\right) \in \mathbb{R}_{\geq 0}^{n},
$$

where $e^{-\infty}=0$. Let $\left\{p^{(i)}=\left(p_{1}^{(i)}, \ldots, p_{n}^{(i)}\right) \in \mathbb{T}^{n}\right\}_{i=1}^{\infty}$ be a sequence satisfying $\max \left(p_{j}^{(i)} \mid i, j\right)<$ const. Then $\left\{p^{(i)}\right\}$ has a convergent subsequence.

We call the set $\mathbb{T}^{n} \backslash \mathbb{R}^{n}$ the corner after Oda [11].

Similar to constructing a polynomial ring from a ring, one can construct a polynomial semiring from a semiring.

Theorem 2.1 ([3], Theorem 2.1.6) Let $S$ be a semiring with an absorbing zero. Then the set of all polynomials

$$
S[x]:=\left\{\sum_{i=0}^{n} a_{i} x^{i} \mid n \in \mathbb{Z}_{\geq 0}, a_{i} \in S\right\}
$$

is a semiring with an absorbing zero.

Corollary 2.1 $\mathbb{T}\left[x_{1}, \ldots, x_{n}\right]$ is a semiring. We call each element a tropical polynomial, or simply a polynomial.

$$
F=\sum_{i_{1}, \ldots, i_{n} \in \mathbb{Z}_{\geq 0}} a_{i_{1} \ldots i_{n}} x_{1}^{i_{1}} \ldots x_{n}^{i_{n}}
$$

We shall often abbreviate $\left(x_{1}, \ldots, x_{n}\right)$ to $X$ and write each element using multi-index:

$$
F=\sum_{I \in \mathbb{Z}_{\geq 0}^{n}} a_{I} X^{I} \in \mathbb{T}[X]:=\mathbb{T}\left[x_{1}, \ldots, x_{n}\right]
$$

where $X^{I}=x_{1}^{i_{1}} \ldots x_{n}^{i_{n}}$ for $I=\left(i_{1}, \ldots, i_{n}\right)$.

Remark We usually omit the term with the coefficient $-\infty$ (zero of $\mathbb{T}$ ). 
Also we usually do not write the coefficient 0 (identity of $\mathbb{T}$ ). Thus for example,

$$
\begin{aligned}
0 & \oplus-\infty x \oplus(-1) x^{2} \oplus 1 x^{3} \oplus 0 x^{4} \oplus-\infty x^{5} \oplus-\infty x^{6} \oplus-\infty x^{7} \oplus \cdots \\
& =0 \oplus(-1) x^{2} \oplus 1 x^{3} \oplus x^{4}
\end{aligned}
$$

Do not mistake $1 x^{3}$ for $x^{3}$. Sometimes $(-1) x^{2}$ is abbreviated to $-x^{2}$. However, this notation is misleading since $-x^{2}$ is not the additive inverse of $x^{2}$ $\left(-x^{2} \oplus x^{2}=x^{2}\right)$.

We frequently write $F \otimes G$ as $F G$.

The zero of $\mathbb{T}[X]$ is $-\infty$. Thus the term nonzero means that it is not $-\infty$. E.g., $x \oplus 2$ and 0 are both nonzero elements.

$A$ monomial is an element with every coefficient being $-\infty$ except one (which may also be $-\infty$ ). A binomial is an element with at most two coefficient being nonzero. Thus a monomial is a binomial. We call a binomial which is not a monomial a strict binomial.

Remark The addition of indexes occurs by multiplying two tropical polynomials. This addition is the usual addition and not the tropical addition. For instance,

$$
x^{2} \otimes x^{3}=x^{5}, \quad(x \oplus 1) \otimes(x \oplus(-1))=x^{2} \oplus 1 x \oplus 0 .
$$

Each tropical polynomial $F=\sum a_{I} x^{I}$ defines a continuous function $\mu(F): \mathbb{T}^{n} \rightarrow \mathbb{T}$ by

$$
\mathbb{T}^{n} \ni p=\left(p_{1}, \ldots, p_{n}\right) \mapsto \mu(F)(p):=\sum_{I} a_{I} p^{I} \in \mathbb{T}
$$

Note that

$$
\begin{aligned}
a_{I} p^{I} & =a_{I} \otimes p_{1}^{i_{1}} \otimes p_{2}^{i_{2}} \otimes \cdots \otimes p_{n}^{i_{n}} \\
& =a_{I}+i_{1} \cdot p_{1}+i_{2} \cdot p_{2}+\cdots+i_{n} \cdot p_{n}
\end{aligned}
$$

The thick lines in Figure 1 illustrates the graphs of two polynomials $F=x \oplus 0$ and $F^{\prime}=x(x \oplus 0)=x^{2} \oplus x$. The thin lines in contrast graphs the value of smaller-valued terms. 

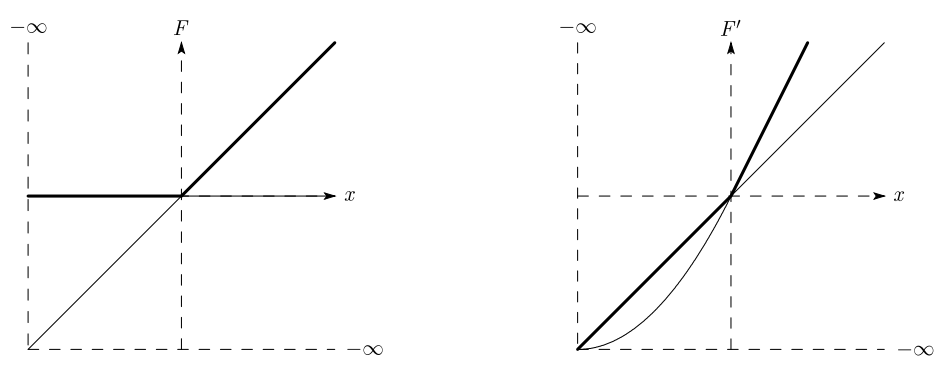

Figure 1. $\quad F=x \oplus 0 / F^{\prime}=x(x \oplus 0)$

Definition 2.2 We call a function $\phi: \mathbb{T}^{n} \rightarrow \mathbb{T}$ a tropical polynomial function (or just a polynomial function) on $\mathbb{T}^{n}$ if there exists a tropical polynomial $F$ such that $\phi=\mu(F)$.

Remark We sometimes identify $F$ and $\mu(F)$. However even if $\mu(F)=$ $\mu(G)$ holds, $F$ and $G$ may be different as tropical polynomials (e.g., $F=$ $x^{2} \oplus 0, G=x^{2} \oplus x \oplus 0$. See Proposition 3.2).

\section{Tropical polynomial functions}

An integral domain has a quotient field.

Definition 3.1 Let $(S,+, \cdot)$ be a semiring. Then an element $a$ of $S$ is a multiplicatively cancellable element if $a b=a c$ infers $b=c$ for any two elements $b$ and $c$.

If every nonzero element of $S$ is a multiplicatively cancellable element, we say that $S$ is multiplicatively cancellative.

Theorem 3.1 ([3], Theorem 2.2.5) Let $(S,+, \cdot)$ be a multiplicatively cancellative semiring. Then there exists a quotient semifield $Q(S)$, that is, a semifield generated by all the elements of $S$ and the multiple inverse of all nonzero elements of $S$ equipped with the following addition/multiplication:

- $F_{1} G_{1}^{-1}+F_{2} G_{2}^{-1}=\left(F_{1} G_{2}+F_{2} G_{1}\right)\left(G_{1} G_{2}\right)^{-1}$,

- $F_{1} G_{1}^{-1} \cdot F_{2} G_{2}^{-1}=\left(F_{1} F_{2}\right)\left(G_{1} G_{2}\right)^{-1}$.

As usual, we often write $F G^{-1}$ as $F / G$.

Remark Every semifield $F$ is multiplicatively cancellative. Thus we can take the quotient $Q(F)$, which equals $F$. So we often write $a b^{-1} \in F$ as $a / b$. 
Thus we are led to the following question: Is $\mathbb{T}[X]$ multiplicatively cancellative?

Proposition 3.1 $\mathbb{T}[X]$ is not multiplicatively cancellative. Indeed a nonzero element $F \in \mathbb{T}[X]$ is a multiplicatively cancellative element if and only if $F$ is a monomial.

Proof. Obviously nonzero monomials are multiplicatively cancellative. Suppose $F$ is not a monomial and take a grading which makes $F$ inhomogeneous. Then divide $F$ into two polynomials $G_{1}$ and $G_{2}$ such that the lowest degree of $G_{1}$ is $d_{1}$ and the highest degree of $G_{2}$ is $d_{2}\left(d_{1}>d_{2}\right)$.

The following equation holds:

$$
\begin{aligned}
\left(G_{1} \oplus G_{2}\right)\left(G_{1}^{2} \oplus G_{1} G_{2} \oplus G_{2}^{2}\right) & =G_{1}^{3} \oplus G_{1}^{2} G_{2} \oplus G_{1} G_{2}^{2} \oplus G_{2}^{3} \\
& =\left(G_{1} \oplus G_{2}\right)\left(G_{1}^{2} \oplus G_{2}^{2}\right) .
\end{aligned}
$$

Since $G_{1}^{2} \oplus G_{1} G_{2} \oplus G_{2}^{2}$ contains the degree $\left(d_{1}+d_{2}\right)$ term which is not contained in $G_{1}^{2} \oplus G_{2}^{2}$, we come to the conclusion.

So we cannot simply take the quotient of $\mathbb{T}[X]$.

Definition 3.2 We say that $F$ and $G$ are equivalent and write as $F \sim G$ when $F(p)=G(p)$ holds for every $p \in \mathbb{T}^{n}$. This is an equivalent relation and we denote $\mathbb{T}[X] / \sim \operatorname{as} \operatorname{Poly}\left(\mathbb{T}^{n}\right)$. $\operatorname{Poly}\left(\mathbb{T}^{n}\right)$ is canonically equipped with tropical operators and is a semiring.

Definition $3.3([2],[10]) \quad$ For a tropical polynomial $F=\sum_{I \in \mathcal{I}} a_{I} X^{I} \in$ $\mathbb{T}[X]$, we define the extended polyhedral domain as

$$
\tilde{\Delta}(F)=\text { ConvexHull }\left\{(I, b) \in \mathbb{R}^{n} \times \mathbb{R} \mid I \in \mathcal{I}, b \leq a_{I}\right\}
$$

\section{Proposition 3.2}

$$
F \sim G \Longleftrightarrow \tilde{\Delta}(F)=\tilde{\Delta}(G)
$$

Proof. Suppose $\tilde{\Delta}(F) \nsubseteq \tilde{\Delta}(G)$ holds and take a point $(I, b)$ from $\tilde{\Delta}(F) \backslash$ $\tilde{\Delta}(G)$. Then it is well-known that there is "a separating hyperplane" $H$ (which separates $\tilde{\Delta}(G)$ and $(I, b)$ ). Let $x_{1}, \ldots, x_{n}$, and $y$ be the coordinates of $\mathbb{R}^{n} \times \mathbb{R}$. Then since $\tilde{\Delta}(G) \subset\{y<M\}$ holds for some constant $M$, we may assume $H$ to be defined as follows: 


$$
p_{1} x_{1}+\cdots+p_{n} x_{n}+y=c .
$$

Then for a point $p=\left(p_{1}, \ldots, p_{n}\right) \in \mathbb{T}^{n}$, we have $F(p)>c>G(p)$.

Conversely suppose that a point $p=\left(p_{1}, \ldots, p_{n}\right)$ satisfies $F(p)>G(p)$. Then by setting $H$ to be

$$
H=\left\{p_{1} x_{1}+\cdots+p_{n} x_{n}+y=F(p)\right\},
$$

we have $H \cap \tilde{\Delta}(F) \neq \emptyset$ and, on the other hand, $H \cap \tilde{\Delta}(G)=\emptyset$.

Corollary 3.1 If $F$ and $G$ are equivalent and $F$ is a monomial, then $F=G$ holds.

Definition 3.4 We call an element of Poly $\left(\mathbb{T}^{n}\right)$ a monomial if a representative element is a monomial. We call an element of Poly $\left(\mathbb{T}^{n}\right)$ a binomial if there exists a binomial representative. Also by a strict binomial we mean a nonmonomial binomial of Poly $\left(\mathbb{T}^{n}\right)$.

Proposition 3.3 Poly $\left(\mathbb{T}^{n}\right)$ is a multiplicatively cancellative semiring.

Proof. Suppose that the elements $F \neq-\infty, G_{1}$, and $G_{2}$ of $\mathbb{T}[X]$ satisfy $F G_{1}=F G_{2}$. For a point $p \in \mathbb{R}^{n}, F(p)$ is not $-\infty$. Thus we have $G_{1}(p)=$ $G_{2}(p)$. Otherwise take $\epsilon$ from $\mathbb{R}=\mathbb{T}^{\times}$and put $\underline{\epsilon}=(\epsilon, \ldots, \epsilon)$. Then $p \oplus \underline{\epsilon}$ is an element of $\mathbb{R}^{n}$. So we have $G_{1}(p \oplus \underline{\epsilon})=G_{2}(p \oplus \underline{\epsilon})$. Since tropical polynomial functions are continuous, we have $G_{1}(p)=G_{2}(p)$ by $\epsilon \rightarrow-\infty$.

Remark Obviously each polynomial $f \in \operatorname{Poly}\left(\mathbb{T}^{n}\right)$ uniquely determines a tropical function by the isomorphism of semirings

$$
\operatorname{Poly}\left(\mathbb{T}^{n}\right) \ni f \mapsto \mu(F) \in\left\{\text { tropical polynomial functions on } \mathbb{T}^{n}\right\},
$$

where $F$ is a representative element. Thus we freely identify an element of $\operatorname{Poly}\left(\mathbb{T}^{n}\right)$ and a polynomial function and write as $f: \mathbb{T}^{n} \rightarrow \mathbb{T}$. Especially we call each element of Poly $\left(\mathbb{T}^{n}\right)$ a (tropical) polynomial function.

\section{Irreducible binomials}

The following equation holds as tropical polynomial functions. 


\section{Lemma 4.1}

$$
\left(f_{1} \oplus \cdots \oplus f_{s}\right)^{k}=f_{1}^{k} \oplus \cdots \oplus f_{s}^{k} \in \operatorname{Poly}\left(\mathbb{T}^{n}\right)
$$

Proof. By expanding the left side, we have

$$
\left(f_{1} \oplus \cdots \oplus f_{s}\right)^{k}=\sum_{\substack{k_{1}, \ldots, k_{s} \in \mathbb{Z}_{\geq 0} \\ k_{1}+\cdots+k_{s}=k}} f_{1}^{k_{1}} \otimes \cdots \otimes f_{s}^{k_{s}} .
$$

For a point $p \in \mathbb{T}^{n}, f_{i}(p) \leq \max \left(f_{1}(p), \ldots, f_{s}(p)\right)$ holds. So we have

$$
f_{1}^{k_{1}}(p) \otimes \cdots \otimes f_{s}^{k_{s}}(p) \leq \max \left(f_{1}^{k}(p), \ldots, f_{s}^{k}(p)\right)=f_{1}^{k}(p) \oplus \cdots \oplus f_{s}^{k}(p),
$$

and thus

$$
\left(f_{1}(p) \oplus \cdots \oplus f_{s}(p)\right)^{k}=f_{1}^{k}(p) \oplus \cdots \oplus f_{s}^{k}(p) .
$$

Definition 4.1 A polynomial $f$ is irreducible if any representative of $f$ cannot be written as a product of two nonconstant polynomials.

Example 1 A nonconstant monomial $\theta$ is irreducible if and only if $\theta=a x_{r}$ for some $a \in \mathbb{R}$ and $r \in\{1, \ldots, n\}$.

For $L=\left(l_{1}, \ldots, l_{n}\right) \in \mathbb{Z}^{n}$, let us define $\operatorname{gcd}(L)$ to be

$$
\operatorname{gcd}(L):=\operatorname{gcd}\left(l_{1}, \ldots, l_{n}\right)>0 .
$$

We say that $L$ is primitive if $\operatorname{gcd}(L)=1$ holds.

Proposition 4.1 Let $\theta=a X^{I} \oplus b X^{J}$ be a strict binomial of $\operatorname{Poly}\left(\mathbb{T}^{n}\right)$, where $I=\left(i_{1}, \ldots, i_{n}\right)$ and $J=\left(j_{1}, \ldots, j_{n}\right)$. Then $\theta$ is irreducible if and only if the following holds:

(i ) for each $r \in\{1, \ldots, n\}$, either $i_{r}$ or $j_{r}$ equals 0 ,

(ii) $I+J$ is primitive.

Remark From (i), we can replace the condition (ii) to the primitiveness of $I-J$.

Proof. Suppose $\theta$ is irreducible. Then obviously (i) holds. Also if $\operatorname{gcd}(I+$ $J)=k \geq 2$ holds, then both $I / k$ and $J / k$ are the elements of $\mathbb{Z}_{\geq 0}^{n}$ and 
Lemma 4.1 shows that $\theta$ can be written as $\theta=\left(\sqrt[k]{a} X^{I / k} \oplus \sqrt[k]{b} X^{I / k}\right)^{k}$. Here, $\sqrt[k]{a}$ is the tropical expression of the usual division of $a$ by $k$, namely, " $a / k "$.

Now searching for a contradiction, suppose $\theta$ satisfies both (i) and (ii) and can be decomposed into a product of two polynomials $\theta_{1}$ and $\theta_{2}$. We may assume that neither $\theta_{1}$ nor $\theta_{2}$ is a monomial. Since the extended polyhedral domain of $\theta$ is a line segment, the extended polyhedral domains of $\theta_{1}$ and $\theta_{2}$ are both line segments. Thus we can write $\theta_{1}$ and $\theta_{2}$ as

$$
\theta_{1}=a_{1} X^{I_{1}} \oplus b_{1} X^{J_{1}}, \quad \theta_{2}=a_{2} X^{I_{2}} \oplus b_{2} X^{J_{2}} .
$$

Then (the representative of) $\theta$ consists of four terms with the indexes being $I_{1}+I_{2}, I_{1}+J_{2}, J_{1}+I_{2}$, and $J_{1}+J_{2}$. Let us put $L_{1}, L_{2} \in \mathbb{Z}^{n}$ as $L_{1}=I_{1}-J_{1}, L_{2}=I_{2}-J_{2}$. Then $L_{1}+L_{2}, L_{1}, L_{2}$, and $\mathbf{0}$ must be on a line. Thus there exists coprime integers $k_{1}, k_{2}$ such that $k_{1} L_{1}=k_{2} L_{2}$. By exchanging $I_{2}$ for $J_{2}$ if necessary, we may assume both $k_{1}$ and $k_{2}$ to be positive. Then we can write $\theta$ as

$$
\theta=a_{1} a_{2} X^{I_{1}+I_{2}} \oplus b_{1} b_{2} X^{J_{1}+J_{2}}
$$

and we have $\operatorname{gcd}(I-J)=\operatorname{gcd}\left(L_{1}+L_{2}\right)$. On the other hand, $k_{1}\left(L_{1}+L_{2}\right)=$ $\left(k_{1}+k_{2}\right) L_{2}$ holds. So $L_{1}+L_{2}$ can be divided by $k_{1}+k_{2} \geq 2$. A contradiction.

Corollary 4.1 A representative of an irreducible binomial is unique.

Example 2 An element $f$ of $\operatorname{Poly}(\mathbb{T})$ is an irreducible binomial if and only if $f=a x \oplus b$ holds, where $a, b$ are the elements of $\mathbb{T}$.

In Section 8, we define a weight of a facet with the help of irreducible binomials.

\section{Rational functions}

Since Poly $\left(\mathbb{T}^{n}\right)$ is multiplicatively cancellative, we can take the quotient.

Definition 5.1 We write the quotient semifield of $\operatorname{Poly}\left(\mathbb{T}^{n}\right)$ as $\operatorname{Rat}\left(\mathbb{T}^{n}\right)$ and call each element a (tropical) rational expression.

Remark Let $h$ be an element of $\operatorname{Rat}\left(\mathbb{T}^{n}\right)$. Then $h$ can be written as 
$h=f / g$, where $f$ and $g$ are elements of $\operatorname{Poly}\left(\mathbb{T}^{n}\right)$.

If both $f$ and $g$ can be taken as a monomial, we call $h$ a monomial. A monomial can be written as $a X^{L}$ for some $a \in \mathbb{T}$ and $L \in \mathbb{Z}^{n}$.

Let $\overline{\mathbb{T}}$ denote the set $\mathbb{T} \cup\{\infty\}$. Then $\overline{\mathbb{T}}$ can be obtained by gluing two $\mathbb{T}^{\prime}$ s along $\mathbb{T}^{\times}$by

$$
\mathbb{T}^{\times} \ni a \mapsto a^{-1} \in \mathbb{T}^{\times}
$$

We induce a topology in $\overline{\mathbb{T}}$ from this construction. In particular, $\overline{\mathbb{T}}$ is compact and sequentially compact.

Since $\overline{\mathbb{T}}=\mathbb{T} \cup\{\infty\}$ canonically contains $\mathbb{T}$, we can calculate the tropical addition and multiplication of elements $a, b$ in $\overline{\mathbb{T}}$ if both $a, b$ are in $\mathbb{T}$. Also we can calculate the inverse $a^{-1}$ if $a$ is in $\mathbb{T}^{\times}$. Let us extend these operations to $\overline{\mathbb{T}}$.

We naturally put $( \pm \infty)^{-1}=\mp \infty$ since $\lim _{a \rightarrow \pm \infty} a^{-1}=\mp \infty$. Thus the inverse element $a^{-1}$ of $\overline{\mathbb{T}}$ is defined for all $a$. Similarly, both $\infty \oplus a$ and $a \oplus \infty$ are defined as $\infty$, and $\infty \otimes a$ and $a \otimes \infty$ are defined as $\infty$ if $a \neq-\infty$. Thus the addition $a \oplus b$ is defined for all $a, b \in \overline{\mathbb{T}}$ and the multiplication $a \otimes b$ is defined for all $a, b \in \overline{\mathbb{T}}$ except for $(a, b)=( \pm \infty, \mp \infty)$.

Let $h=f / g$ be an element of $\operatorname{Rat}\left(\mathbb{T}^{n}\right)$. Note that both $f(p)$ and $g(p)$ are elements of $\mathbb{T}$ for $p \in \mathbb{T}^{n}$. Thus for every $p \in \mathbb{T}^{n}$ outside $f(p)=g(p)=-\infty$, $\mu(h)(p):=f(p) / g(p)=f(p) \otimes g(p)^{-1}$ is an element of $\overline{\mathbb{T}}=\mathbb{T} \cup\{\infty\}$.

Thus we have a continuous function

$$
\left\{p \in \mathbb{T}^{n} \mid f(p) \oplus g(p) \neq-\infty\right\} \ni p \mapsto \mu(h)(p) \in \overline{\mathbb{T}}
$$

which is at least defined over $\mathbb{R}^{n}$. We will write the function above as

$$
\mu(h): \mathbb{T}^{n} \rightarrow \overline{\mathbb{T}}
$$

to imply that the closure of the domain is $\mathbb{T}^{n}$.

Definition 5.2 Let $\phi: S \rightarrow \overline{\mathbb{T}}$ be a function with $S$ being a subset of $\mathbb{T}^{n}$ containing $\mathbb{R}^{n}$. We call $\phi$ a (tropical) rational function if there exists a rational expression $h$ such that $\phi=\mu(h)$. Rational functions are canonically equipped with tropical operators and forms a semifield.

Remark We do not distinguish a rational expression from a rational func- 
tion since they are obviously isomorphic as semifields. We also call each element of $\operatorname{Rat}\left(\mathbb{T}^{n}\right)$ a rational function and write as $h: \mathbb{T}^{n} \rightarrow \overline{\mathbb{T}}$ by abbreviating $\mu(h)(p)$ to $h(p)$.

Remark A function taking the value $\infty$ everywhere is not a rational function since $0 /(-\infty) \notin \operatorname{Rat}\left(\mathbb{T}^{n}\right)$.

Remark If $h$ is nonzero, $\operatorname{im}\left(\left.h\right|_{\mathbb{R}^{n}}\right) \subset \mathbb{R}$.

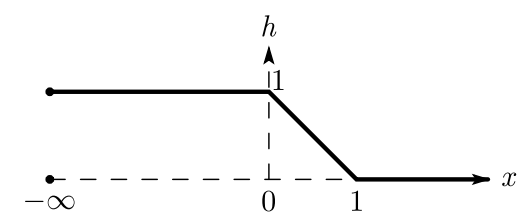

Figure 2. $h=(x \oplus 1) /(x \oplus 0)$

Figure 2 graphs a rational function $h=(x \oplus 1) /(x \oplus 0)$. It is not convex around $x=0$. This is the main difference between a graph of a polynomial function and that of a rational function. See Corollary 9.2 and Corollary 9.4 .

Remark A polynomial function is a rational function with the domain being $\mathbb{T}^{n}$ and the image contained in $\mathbb{T}$.

\section{Meromorphic functions}

Also in tropical geometry, a rational function is a meromorphic function.

Definition 6.1 Let $D$ be a subset of $\mathbb{T}^{n}$. We call a function $\phi: D \rightarrow \overline{\mathbb{T}}$ a piecewise linear function if there exists a covering $\left\{D_{\lambda}\right\}_{\lambda \in \Lambda}$ of $D$ such that $\left.\phi\right|_{D_{\lambda}}=\left.m_{\lambda}\right|_{D_{\lambda}}$ for each $D_{\lambda}$, where each $m_{\lambda} \in \operatorname{Rat}\left(\mathbb{T}^{n}\right)$ is a monomial. In particular, if we can take a finite covering, we call $\phi$ a piecewise linear function of finite type.

Remark We take the covering $\left\{D_{\lambda}\right\}$ to be minimal provided that each $D_{\lambda}$ is connected. Thus if $D_{\lambda_{1}} \cap D_{\lambda_{2}} \neq \emptyset$ holds, then $\left.\phi\right|_{D_{\lambda_{2}}} \neq\left. m_{\lambda_{1}}\right|_{D_{\lambda_{2}}}$.

Definition 6.2 Let $\phi: D \rightarrow \overline{\mathbb{T}}$ be a piecewise linear function with $D$ being an open dense subset of $\mathbb{T}^{n}$. Then $\phi$ is called a (tropical) meromorphic function if it satisfies the followings: 
- $\phi$ is continuous,

- $\operatorname{im} \phi \neq\{\infty\}$,

- $\left\{D_{\lambda}\right\}$ is locally finite; For every $p \in \mathbb{T}^{n}$, there exists a neighborhood $B$ such that

$$
\#\left\{\lambda \in \Lambda \mid B \cap D_{\lambda} \neq \emptyset\right\}<\infty .
$$

A meromorphic function $\phi$ is of finite type if $\phi$ is of finite type as a piecewise linear function. Meromorphic functions are also equipped with tropical operators and forms a semifield.
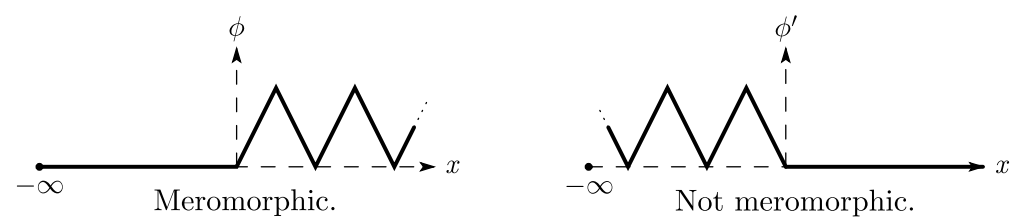

Figure 3. Functions $\phi$ and $\phi^{\prime}$

Figure 3 graphs two functions $\phi$ and $\phi^{\prime}$. $\phi$ is a meromorphic function not of finite type. $\phi^{\prime}$ is not a meromorphic function because the numbers of $D_{\lambda}$ 's are infinite around $x=-\infty$.

Remark We say that two meromorphic functions $\phi_{1}: D_{1} \rightarrow \mathbb{T}$ and $\phi_{2}:$ $D_{2} \rightarrow \mathbb{T}$ are equivalent if $\left.\phi_{1}\right|_{D_{1} \cap D_{2}}=\left.\phi_{2}\right|_{D_{1} \cap D_{2}}$ holds. Then this is an equivalence relation since a meromorphic function is continuous.

Thus from now on, we mean by a meromorphic function an equivalent class or more precisely, a representative element with the domain $D$ being maximal. Especially, every meromorphic function is defined over $\mathbb{R}^{n}$.

Example 3 Let $\phi$ be a meromorphic function satisfying $\# \Lambda=2$. Then either $\phi=m_{1} \oplus m_{2}$ or $\phi=\left(m_{1}^{-1} \oplus m_{2}^{-1}\right)^{-1}=m_{1} m_{2} /\left(m_{1} \oplus m_{2}\right)$.

We call a point in $\mathbb{T}^{n} \backslash D$ a point of indeterminacy. Let $p$ be a point of indeterminacy. Then $\lim _{i \rightarrow \infty} \phi\left(p_{i}\right)$ varies (if exists) by the choice of an infinite sequence $\left\{p_{i} \in D\right\}_{i=1}^{\infty}$ converging to $p$.

Example 4 A rational function $\phi=(x \oplus 1 y) /(x \oplus y)$ has a point of indeterminacy at $p=(-\infty,-\infty)$. Let $T$ be the set of sequences $\left\{p_{i} \in\right.$ $\left.\mathbb{T}^{2} \backslash\{p\}\right\}_{i=1}^{\infty}$ such that 
- $\left\{p_{i}\right\}$ converges to $p$,

- $\left\{\phi\left(p_{i}\right)\right\}$ converges.

Then

$$
\left\{\lim _{i \rightarrow \infty} \phi\left(p_{i}\right) \mid\left\{p_{i}\right\} \in T\right\}=[0,1] .
$$

\section{Tropical hypersurfaces}

From now on, we will treat geometry of meromorphic functions on $\mathbb{T}^{n}$. We denote by + and - the usual addition and multiplication. Do not mistake them for tropical addition/multiplication. In fact, we hardly use the tropical operators except for describing tropical functions.

First, we remark some definitions and properties of a convex function ([13]).

Definition 7.1 Let $D$ be a convex subset of $\mathbb{R}^{n}$. We say that a function $\Phi: D \rightarrow \mathbb{R}$ is a convex function if the epigraph of $f$ defined as

$$
\{(x, \mu) \mid x \in D, \mu \in \mathbb{R}, \mu \geq \Phi(x)\}
$$

is a convex subset of $\mathbb{R}^{n+1}$.

If $-\Psi$ is a convex function, we call $\Psi$ a concave function.

Theorem 7.1 ([13], Theorem 4.1) $\Phi$ is convex if and only if

$$
\Phi((1-\lambda) x+\lambda y) \leq(1-\lambda) \Phi(x)+\lambda \Phi(y), \quad 0<\lambda<1
$$

holds for every $x, y \in D$.

Definition 7.2 We say that $\Phi$ is locally convex at a point $p \in D$ if there exists an open neighborhood $B$ of $p$ such that $\left.\Phi\right|_{B}$ is a convex function.

Proposition 7.1 Suppose $\Phi$ is continuous. Then

$\Phi$ is a convex function $\Leftrightarrow \Phi$ is locally convex at every point.

Proof. $\Rightarrow$ is obvious. $\Leftarrow$ comes from the compactness of $[0,1]$.

Let $\phi$ be a meromorphic function defined over $D$ by $\left.\phi\right|_{D_{\lambda}}=\left.m_{\lambda}\right|_{D_{\lambda}}$ for each $\lambda \in \Lambda$. Put three subsets $V^{o}(\phi), Z(\phi)$, and $P(\phi)$ of $D$ as follows: 


$$
\begin{array}{rlr}
V^{o}(\phi) & :=\bigcup_{\substack{\lambda_{1}, \lambda_{2} \in \Lambda \\
\lambda_{1} \neq \lambda_{2}}} D_{\lambda_{1}} \cap D_{\lambda_{2}}, \quad \text { the corner locus of } \phi, \\
Z(\phi) & :=\{p \in \mathbb{T} \mid \phi(p)=-\infty\}, \quad \text { the zero locus of } \phi, \\
P(\phi) & :=\{p \in \mathbb{T} \mid \phi(p)=\infty\}, \quad \text { the pole locus of } \phi .
\end{array}
$$

Remark $P(\phi)$ is contained in the corner $\mathbb{T}^{n} \backslash \mathbb{R}^{n}$. Also $Z(\phi)$ is contained in the corner except for $\phi \neq-\infty$.

Definition 7.3 For a meromorphic function $\phi$, we define the mixed loci $V(\phi)$ of $\phi$ to be

$$
V(\phi):=V^{o}(\phi) \cup Z(\phi) \cup P(\phi)
$$

We call $\phi$ a defining (meromorphic) function of $V(\phi)$.

Example 5 Let $a$ be a constant function. Then

$$
V(a)= \begin{cases}\mathbb{T}^{n}, & \text { if } a=-\infty \\ \emptyset, & \text { otherwise }\end{cases}
$$

The inverse also holds:

$$
\left\{\begin{array}{l}
V(\phi)=\mathbb{T}^{n} \quad \Rightarrow \quad \phi=-\infty \\
V(\phi)=\emptyset \quad \Rightarrow \quad \phi \text { is a nonzero constant. }
\end{array}\right.
$$

Remark $V(\phi)=V\left(\phi^{k}\right)$ holds for any positive integer $k$.

On each connected component of $D \backslash V(\phi), \phi$ is a monomial. We call each connected component an open chamber and its closure in $D$ a chamber. A chamber equals a $D_{\lambda}$. The number of chambers is finite if $\phi$ is of finite type.

Suppose $\left.\phi\right|_{D_{\lambda}}=\left.a X^{L}\right|_{D_{\lambda}}$ holds. We call $L$ the exponent of $D_{\lambda}$. Since $\left.\phi^{k}\right|_{D_{\lambda}}=\left.a^{k} X^{k \cdot L}\right|_{D_{\lambda}}$ holds, the exponent of $D_{\lambda}$ of $\phi^{k}$ is $k \cdot L$.

Let $f$ be an element of $\operatorname{Poly}\left(\mathbb{T}^{n}\right)$. Note that $P(f)$ is an empty set.

Definition 7.4 The mixed loci of a nonconstant polynomial function is called a tropical hypersurface. 

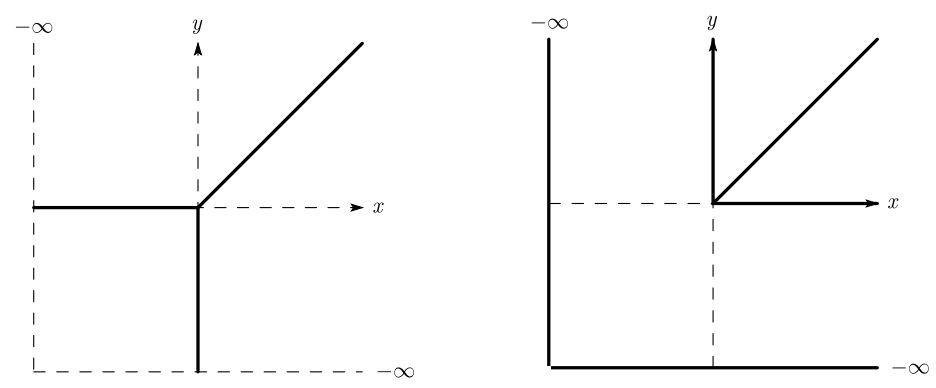

Figure 4. Hypersurface (line) / non-hypersurface

Example 6 The left figure of Figure 4 is the hypersurface defined by $x \oplus y \oplus 0$. Since the defining polynomial is linear, $V(x \oplus y \oplus 0)$ is "a line". On the other hand, the right figure is the mixed loci given by $V\left(x y^{-1}(x \oplus\right.$ $\left.0)^{-1}(y \oplus 0)^{-1}(x \oplus y \oplus 0)\right)$. Note that the defining function has a point of indeterminacy at $(-\infty,-\infty)$. This is not a hypersurface. See Example 7.

\section{Remark}

$$
V(f g)=V(f) \cup V(g), \quad{ }^{\forall} f, g \in \operatorname{Poly}\left(\mathbb{T}^{n}\right)
$$

holds. However, $V^{o}(f g)$ may strictly contain $V^{o}(f) \cup V^{o}(g)$ if either $f$ or $g$ is a monomial (e.g., $V^{o}(x(x \oplus 0))=V^{o}\left(x^{2} \oplus x\right)=\{-\infty, 0\}, V^{o}(x) \cup V^{o}(x \oplus 0)=$ $\{0\})$. This is one of the reasons why we should consider the zero locus (and the pole locus) along with the corner locus.

The definition of a tropical hypersurface is the same as the traditional definition provided $f$ is not a monomial.

Proposition 7.2 Let $F=\sum_{I \in \mathcal{I}} a_{I} X^{I} \in \mathbb{T}[X]$ be a polynomial satisfying $\# \mathcal{I} \geq 2$ by allowing a coefficient to be $-\infty$ if necessary. Then

$$
V(F)=\left\{p \in \mathbb{T}^{n} \mid F(p)=a_{J_{1}} p^{J_{1}}=a_{J_{2}} p^{J_{2}} \text { for some } J_{1}, J_{2} \in \mathcal{I}, J_{1} \neq J_{2}\right\} .
$$

Proof. Let us put $V$ to be the right hand side of the above.

Since both $V^{o}(F)$ and $Z(F)$ are contained in $V$, we have $V(F) \subset V$.

Conversely, suppose $p$ is a point in $V$. If $F$ takes the value $-\infty$ at $p$, then $p$ is in $Z(F)$. Otherwise $F$ is not a monomial and $p$ is contained in the corner locus. 


\section{The weight of a facet}

In this section, we mean by a meromorphic function a meromorphic function satisfying $\# \Lambda \geq 2$.

The tropical hypersurface $V(\theta)$ of an irreducible binomial $\theta$ is as follows:

- If $\theta=a x_{r}$ is a monomial, then $V(\theta)=\left\{\left(p_{1}, \ldots, p_{n}\right) \in \mathbb{T}^{n} \mid p_{r}=-\infty\right\}$,

- If $\theta=a X^{I} \oplus b X^{J}\left(I=\left(i_{1}, \ldots, i_{n}\right), J=\left(j_{1}, \ldots, j_{n}\right)\right)$ is a strict binomial, then $V(\theta)$ is the closure of an affine hyperplane in $\mathbb{R}^{n}$ defined by

$$
\left(i_{1}-j_{1}\right) x_{1}+\cdots+\left(i_{n}-j_{n}\right) x_{n}=a-b .
$$

Note that the following holds.

Proposition 8.1 Let $\theta$ and $\theta^{\prime}$ be irreducible binomials satisfying $V(\theta)=$ $V\left(\theta^{\prime}\right)$. Then $\theta=\theta^{\prime}$ holds up to constant.

Proof. The statement is obvious if $\theta$ (and thus $\theta^{\prime}$ too) are monomials. So we may assume that both $\theta$ and $\theta^{\prime}$ are strict binomials and write them down as

$$
\theta=a X^{I} \oplus b X^{J}, \quad \theta^{\prime}=a^{\prime} X^{I^{\prime}} \oplus b^{\prime} X^{J^{\prime}}
$$

Then $V(\theta)=V\left(\theta^{\prime}\right)$ and the primitiveness of $I-J$ and $I^{\prime}-J^{\prime}$ yields either $I-J=I^{\prime}-J^{\prime}$ or $I-J=J^{\prime}-I^{\prime}$. In the first case, we have $I=I^{\prime}$ and $J=J^{\prime}$. Then $a-b=a^{\prime}-b^{\prime}$ holds. In the second case, we have $I=J^{\prime}$ and $J=I^{\prime}$. Then we have $a-b=b^{\prime}-a^{\prime}$.

Definition 8.1 Let $\phi$ be a meromorphic function defined at $D \subset \mathbb{T}^{n}$ and put $V^{\prime}$ to be a subset of $V(\phi)$ given by

$$
V^{\prime}=\left(V(\phi) \backslash V^{o}(\phi)\right) \cup\left\{p \in \mathbb{R}^{n} \mid p \in D_{\lambda_{1}} \cap D_{\lambda_{2}}, \quad \exists !\left\{\lambda_{1}, \lambda_{2} \mid \lambda_{1} \neq \lambda_{2}\right\} \subset \Lambda\right\}
$$

(which corresponds to all the "smooth" points of $V(\phi)$ ).

Then we define as follows:

- an open facet is a connected component of $V^{\prime}$,

- a facet (or a $(n-1)$-cell) is the closure of an open facet,

- a cornered (resp. a non-cornered) facet is a facet contained (resp. not contained) in the corner $\mathbb{T}^{n} \backslash \mathbb{R}^{n}$, 
- an intersecting neighborhood $B$ of a facet $H$ is an open subset of $D$ satisfying $\emptyset \neq B \cap V(\phi) \subset H$.

We write the set of facets (resp. non-cornered facets) as $\mathcal{L}(\phi)\left(\right.$ resp. $\left.\mathcal{L}_{\mathbb{R}}(\phi)\right)$.

Remark From the openness of $B, B \cap V(\phi)$ is contained in an open facet.

Let $\phi$ be a meromorphic function and take a facet $H$ of $\phi$. Then there exists an irreducible binomial $\theta$ satisfying $V(\theta) \supset H$. Note that $\theta$ is unique up to constant by Proposition 8.1.

Lemma 8.1 There exists a unique integer $w(H)$ and a monomial $m \in$ $\operatorname{Rat}\left(\mathbb{T}^{n}\right)$ satisfying $V(m) \cap B=\emptyset$ such that

$$
\left.\phi\right|_{B}=\left.m \otimes \theta^{w(H)}\right|_{B}
$$

Remark This $w(H)$ is independent of the choice of $\theta$.

Proof. If $H$ is cornered, then the statement is obvious. Thus suppose $H$ is non-cornered. Let $D_{1}, D_{2}$ be the chambers of $\phi$ adjacent to $H$ and put

$$
\left.\phi\right|_{B}=\left\{\begin{array}{ll}
a X^{L_{1}}, & \text { if } X \in D_{1} \\
b X^{L_{2}}, & \text { if } X \in D_{2}
\end{array}, \quad \theta=a^{\prime} X^{I^{\prime}} \oplus b^{\prime} X^{J^{\prime}}\right.
$$

where we assume $\left.\theta\right|_{D_{1}}=a^{\prime} X^{I^{\prime}}$ and $\left.\theta\right|_{D_{2}}=b^{\prime} X^{J^{\prime}}$. Then either $\left.\phi\right|_{B}=$ $a X^{L_{1}} \oplus b X^{L_{2}}$ or $\left.\phi\right|_{B}=a b X^{L_{1}+L_{2}} /\left(a X^{L_{1}} \oplus b X^{L_{2}}\right)$ holds. It is enough to show the case on $\left.\phi\right|_{B}=a X^{L_{1}} \oplus b X^{L_{2}}$. Here, $a, b, a^{\prime}$, and $b^{\prime}$ are all nonzero.

Since $V(\phi) \cap B=V(\theta) \cap B$ holds, we have

$$
L_{2}-L_{1}=w(H) \cdot\left(J^{\prime}-I^{\prime}\right) \text { and } b-a=w(H) \cdot\left(b^{\prime}-a^{\prime}\right)
$$

for some integer $w(H)$ from the primitiveness of $J^{\prime}-I^{\prime}$. Let $L$ and $c$ be defined as

$$
L=I-w(H) I^{\prime}=J-w(H) J^{\prime}, \quad c=a-w(H) a^{\prime}=b-w(H) b^{\prime} .
$$

Since $\theta^{w(H)}=\left(a^{\prime}\right)^{w(H)} X^{w(H) \cdot I^{\prime}} \oplus\left(b^{\prime}\right)^{w(H)} X^{w(H) \cdot J^{\prime}}$ holds, we have

$$
\left.\phi\right|_{B}=c X^{L} \otimes \theta^{w(H)} .
$$


Note that $V\left(c X^{L}\right) \cap B$ is an empty set because it is not contained in $V(\theta)$. Thus the statement is proved.

Definition 8.2 We call $w(H)$ the weight of $H$.

Remark Let $H$ be a cornered facet. Then from the definition,

$$
\begin{aligned}
w(H)>0 & \Leftrightarrow H \subset Z(\phi), \\
w(H)<0 & \Leftrightarrow H \subset P(\phi) .
\end{aligned}
$$

In the next section, we will see that the signature gives the local convexity of the function at an intersecting neighborhood if $H$ is non-cornered.

Remark Let $\phi$ be a meromorphic function and $H$ be a facet of weight $w(H)$. Then $H$ is also a facet of $\phi^{k}$ of weight $k \cdot w(H)$.

Corollary 8.1 Suppose $H$ is non-cornered and let the chambers adjacent to $H$ be $D_{\lambda_{1}}$ and $D_{\lambda_{2}}$ with the exponents being $L_{\lambda_{1}}$ and $L_{\lambda_{2}}$ respectively. Also let $v$ be the vector orthogonal to $H$ pointing from $D_{\lambda_{1}}$ to $D_{\lambda_{2}}$. Then

$$
L_{\lambda_{2}}-L_{\lambda_{1}}=w(H) \cdot v
$$

Proof. Evident from the proof of Lemma 8.1.

We call a subset $E$ of $S$ a non-cornered $(n-2)$-face if

- $E$ is given as an intersection of two facets,

- the affine hull of $E \cap \mathbb{R}^{n}$ in $\mathbb{R}^{n}$ (denoted as aff $\left(E \cap \mathbb{R}^{n}\right)$ ) is of codimension two as a subspace of $\mathbb{R}^{n}$.

Lemma 8.2 Let $H$ be a facet satisfying $\operatorname{dim} \operatorname{aff}\left(E \cap H \cap \mathbb{R}^{n}\right)=n-2$, where $E$ is a non-cornered $(n-2)$-face. Then $E \subset H$ holds.

Proof. It is enough to show that for a chamber $D^{\prime}$ satisfying $\operatorname{dim}$ aff $(E \cap$ $\left.D^{\prime} \cap \mathbb{R}^{n}\right)=n-2, E \subset D^{\prime}$ holds. In other words, it is enough to show that the monomial representing $\phi$ at $D^{\prime}$ above also equals $\phi$ at $E$.

Since $E$ is given as an intersection of two facets, there exists a chamber $D$ containing $E$. Let $m, m^{\prime}$ be the monomials satisfying $\left.\phi\right|_{D}=\left.m\right|_{D},\left.\phi\right|_{D^{\prime}}=$ $\left.m^{\prime}\right|_{D^{\prime}}$. Then $\phi(p)=m(p)$ holds for every $p \in E$. Since $m(q)=m^{\prime}(q)$ holds for every point $q \in D \cap D^{\prime}, m(q)=m^{\prime}(q)$ also holds for every point $q$ in the closure $\overline{\operatorname{aff}\left(D \cap D^{\prime} \cap \mathbb{R}^{n}\right)}$. $\overline{\operatorname{aff}\left(D \cap D^{\prime} \cap \mathbb{R}^{n}\right)}$ contains $E$. 
Theorem 8.1 (Balancing property) For a non-cornered $(n-2)$-face $E$ of $\phi$, write the chambers adjacent to $E$ as $D_{1}, \ldots, D_{m}$. Here, we assume $D_{i} \cap D_{j} \supsetneq E$ if and only if $j-i \equiv \pm 1(\bmod m)$. Then put the facet adjacent to $D_{i}$ and $D_{i+1}$ as $H_{i}$ (we assume $D_{m+1}=D_{1}$ ). Also let the primitive vector orthogonal to $H_{i}$ pointing from $D_{i}$ to $D_{i+1}$ be $v_{i}$. Then

$$
\sum_{i=1}^{m} w\left(H_{i}\right) \cdot v_{i}=\mathbf{0}
$$

where the operators are the usual operators and not the tropical ones.

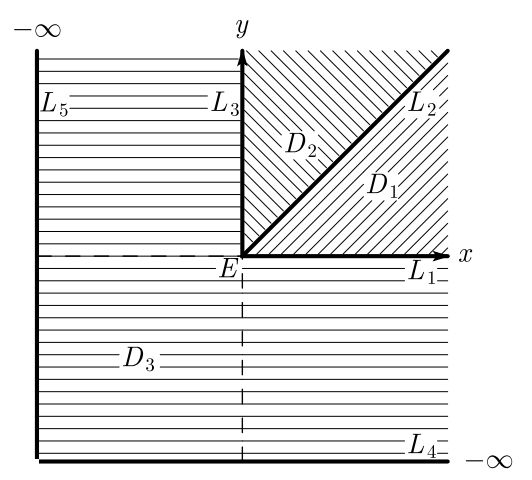

Table I

\begin{tabular}{c|c|c|c}
\hline$i$ & $\left.\phi\right|_{B_{i}}$ & $w\left(L_{i}\right)$ & $v\left(L_{i}\right)$ \\
\hline 1 & $x y^{-1}(y \oplus 0)^{-1}$ & -1 & $(0,1)$ \\
2 & $y^{-2}(x \oplus y)$ & 1 & $(-1,1)$ \\
3 & $x y^{-1}(x \oplus 0)^{-1}$ & -1 & $(-1,0)$ \\
4 & $x y^{-1}$ & 1 & - \\
5 & $x y^{-1}$ & -1 & - \\
\hline
\end{tabular}

Figure 5. Facets and their properties

Example 7 Consider $\phi=x y^{-1}(x \oplus 0)^{-1}(y \oplus 0)^{-1}(x \oplus y \oplus 0)$ as in Example 6 and put the facets $L_{1}, \ldots, L_{5}$ as in Figure 5. Put $B_{i}$ to be an intersecting neighborhood of $L_{i}$. Then the list of $\left.\phi\right|_{B_{i}}, w\left(L_{i}\right)$, and $v\left(L_{i}\right)$ for each $i$ is given by Table I.

Let $E$ be $(0,0)$. The balancing property at $E$ will be

$$
w\left(L_{1}\right) \cdot v_{1}+w\left(L_{2}\right) \cdot v_{2}+w\left(L_{3}\right) \cdot v_{3}=\mathbf{0} .
$$

\section{The geometry of meromorphic functions}

Definition 9.1 We say that a meromorphic function $\phi$ is a convex function (resp. a concave function) if $\left.\phi\right|_{\mathbb{R}^{n}}$ is convex (resp. concave).

Proposition 9.1 Let $\phi$ and $\psi$ be convex meromorphic functions. Then 
both $\phi \oplus \psi$ and $\phi \otimes \psi$ are convex.

Proof. Let $p_{1}, p_{2} \in \mathbb{R}^{n}$ and $t \in[0,1]$. Then

$$
\begin{aligned}
(\phi \oplus \psi)\left(t p_{1}+(1-t) p_{2}\right) & =\max \left(\phi\left(t p_{1}+(1-t) p_{2}\right), \psi\left(t p_{1}+(1-t) p_{2}\right)\right) \\
& \leq \max \left(t \phi\left(p_{1}\right)+(1-t) \phi\left(p_{2}\right), t \psi\left(p_{1}\right)+(1-t) \psi\left(p_{2}\right)\right) \\
& \leq t \max \left(\phi\left(p_{1}\right), \psi\left(p_{1}\right)\right)+(1-t) \max \left(\phi\left(p_{2}\right), \psi\left(p_{2}\right)\right) \\
& =t(\phi \oplus \psi)\left(p_{1}\right)+(1-t)(\phi \oplus \psi)\left(p_{2}\right) .
\end{aligned}
$$

The convexity of $\phi \otimes \psi$ is well-known and also easy to prove.

Corollary 9.1 A polynomial function is convex.

Proposition 9.2 Let $\phi$ be a meromorphic function of finite type defined by $\left.\phi\right|_{D_{\lambda}}=\left.m_{\lambda}\right|_{D_{\lambda}}$ for each chamber $D_{\lambda}$ with $m_{\lambda}$ being a monomial. Then

$$
\phi=\sum_{\lambda \in \Lambda} m_{\lambda} \Longleftrightarrow \phi \text { is convex. }
$$

Proof. From the previous proposition, we already know that $\Rightarrow$ holds.

Suppose $\phi \neq \sum m_{\lambda}$. Then there exists a point $p$ such that $\phi(p)<$ $\sum m_{\lambda}(p)$. Especially, we can take $p$ from $\mathbb{R}^{n}$ from the continuity of $\phi$. Let $\lambda_{1} \in \Lambda$ satisfy $\sum m_{\lambda}(p)=m_{\lambda_{1}}(p)$ and take a point $q$ from the open chamber of $D_{\lambda_{1}} \cap \mathbb{R}^{n}$. Also take $t \in(0,1)$ small enough so that $t p+(1-t) q$ is an element of $D_{\lambda_{1}}$. Then

$$
\begin{aligned}
\phi(t p+(1-t) q) & =m_{\lambda_{1}}(t p+(1-t) q) \\
& =t m_{\lambda_{1}}(p)+(1-t) m_{\lambda_{1}}(q) \\
& >t \phi(p)+(1-t) \phi(q)
\end{aligned}
$$

which yields $\phi$ is not convex.

Lemma 9.1 Let $\phi$ be a meromorphic function defined by

$$
\phi=\left\{\begin{array}{lll}
a_{1} X^{L_{1}}, & \text { if } X \in D_{1} & \left(L_{1} \in \mathbb{Z}^{n}\right), \\
a_{2} X^{L_{2}}, & \text { if } X \in D_{2} & \left(L_{2} \in \mathbb{Z}^{n}\right),
\end{array}\right.
$$


where $D_{1}$ and $D_{2}$ are the nonempty chambers of $\phi$ and put $H$ to be the unique facet. Then

$$
\begin{aligned}
\phi \text { is convex } & \Longleftrightarrow w(H)>0, \\
\phi \text { is concave } & \Longleftrightarrow w(H)<0 .
\end{aligned}
$$

Proof. First of all, we know that $w(H) \neq 0$ from the minimality of $\left\{D_{\lambda}\right\}$.

By sliding $\phi$ if necessary, we may assume both $a_{1}$ and $a_{2}$ to be 0 . Let $v$ be a primitive vector orthogonal to $H$ as before pointing from $D_{1}$ to $D_{2}$. Then from Corollary 8.1, we have $L_{2}-L_{1}=w(H) \cdot v$. Take a point $p$ from $D_{2}$. Then

$$
\phi(p)=p^{L_{2}}=p^{L_{1}+w(H) \cdot v}=p^{L_{1}} \otimes p^{w(H) \cdot v}=p^{L_{1}}+w(H) \cdot p^{v} .
$$

Note that $p^{v} \geq 0$ holds because $p$ is a point in $D_{2}$. Thus we have

$$
\phi(p) \begin{cases}>p^{L_{1}}, & \text { if } w(H)>0, \\ <p^{L_{1}}, & \text { if } w(H)<0,\end{cases}
$$

which proves the statement.

Corollary 9.2 Let $H$ be a non-cornered facet of a meromorphic function $\phi$ and $B$ be an intersecting neighborhood. Also let the exponents of the chambers adjacent to $H$ be $L_{1}$ and $L_{2}$. Then we have the followings:

$$
\begin{aligned}
\left.\phi\right|_{B} \text { is convex } \Leftrightarrow w(H)=\operatorname{gcd}\left(L_{2}-L_{1}\right)>0, \\
\left.\phi\right|_{B} \text { is concave } \Leftrightarrow w(H)=-\operatorname{gcd}\left(L_{2}-L_{1}\right)<0 .
\end{aligned}
$$

Proof. Corollary 8.1 and Lemma 9.1.

Proposition 9.3 Let $\phi$ be a meromorphic function. Then

$$
\phi \text { is convex } \Longleftrightarrow w(H)>0, \quad{ }^{\forall} H \in \mathcal{L}_{\mathbb{R}}(\phi) \text {. }
$$

Proof. We already know that $\Rightarrow$ holds.

If $w(H)>0$ holds for every facets, $\phi$ is locally convex outside boundaries of facets. Thus we come to the conclusion from the continuity of $\phi$ and Proposition 7.1. 
Corollary 9.3 Let $\phi$ be a meromorphic function defined by $\left.\phi\right|_{D_{\lambda}}=\left.m_{\lambda}\right|_{D_{\lambda}}$ for each chamber $D_{\lambda}$, where $m_{\lambda}$ is a monomial. Then

$$
\phi=\sum_{\lambda \in \Lambda} m_{\lambda} \Longleftrightarrow w(H)>0, \quad{ }^{\forall} H \in \mathcal{L}_{\mathbb{R}}(\phi) .
$$

Example $8 \quad V(\phi)$ given in Figure 5 is not a hypersurface restricted to $D=\mathbb{T}^{2} \backslash\{(-\infty,-\infty)\}$ because there are no positive integers $w_{1}, w_{2}, w_{3}$ satisfying the balancing property at $E$ :

$$
w_{1} \cdot v_{1}+w_{2} \cdot v_{2}+w_{3} \cdot v_{3} \neq \mathbf{0} \text { if } w_{1}, w_{2}, w_{3} \in \mathbb{Z}_{>0} .
$$

Corollary 9.4 Let $\phi$ be a meromorphic function of finite type. Then $\phi$ is an element of $\operatorname{Poly}\left(\mathbb{T}^{n}\right)$ if and only if every weight of facets is positive.

Proof. From the Corollary 9.3, we know that $\phi=\sum m_{\lambda}$ holds. If any of $m_{\lambda}$ is not contained in Poly $\left(\mathbb{T}^{n}\right)$, the pole locus exists. The weight of a facet contained in a pole locus is always negative. The other side is a consequence of Corollary 9.3 and the fact that polynomial functions does not have a pole locus.

Let $H$ be a non-cornered facet of $\phi$ of negative weight. Assume $\phi$ satisfies $\left.\phi\right|_{D_{\lambda}}=\left.m_{\lambda}\right|_{D_{\lambda}}$ as before. Let the chambers adjacent to $H$ be $D_{\lambda_{1}}$ and $D_{\lambda_{2}}$. Then by setting a rational function $h$ to be $h=m_{\lambda_{1}} \oplus m_{\lambda_{2}}$, we have $\left.h\right|_{D_{\lambda_{1}}}=\left.m_{\lambda_{2}}\right|_{D_{\lambda_{1}}}$ and $\left.h\right|_{D_{\lambda_{2}}}=\left.m_{\lambda_{1}}\right|_{D_{\lambda_{2}}}$. Also the facet $H^{\prime}$ of $h$ satisfies $w\left(H^{\prime}\right)=-w(H)>0$ and $H^{\prime} \supset H$.

Now $h \otimes \phi$ is a meromorphic function satisfying $\left.h \otimes \phi\right|_{D_{\lambda_{1}}}=m_{\lambda_{1}} \otimes$ $\left.m_{\lambda_{2}}\right|_{D_{\lambda_{1}}}$ and also $\left.h \otimes \phi\right|_{D_{\lambda_{2}}}=\left.m_{\lambda_{1}} \otimes m_{\lambda_{2}}\right|_{D_{\lambda_{2}}}$. Thus $H$ is not a facet of $h \otimes \phi$ anymore. $h \otimes \phi$ may have new facets though their weights are positive.

Let us formulate what we have seen.

Let $\mathcal{L}_{\mathbb{R}}^{-}(\phi)$ be a subset of $\mathcal{L}_{\mathbb{R}}(\phi)$ such that the weight of each facet is negative.

Proposition 9.4 Let $\phi$ be a meromorphic function. Then there exists a rational function $h$ such that

$$
\mathcal{L}_{\mathbb{R}}^{-}(\phi) \supsetneq \mathcal{L}_{\mathbb{R}}^{-}(h \otimes \phi) .
$$

If $\phi$ is of finite type, then the number of facets is finite so we have the 
followings.

Proposition 9.5 Let $\phi$ be a meromorphic function of finite type. Then there exists a rational function $h$ such that

$$
\mathcal{L}_{\mathbb{R}}^{-}(h \otimes \phi)=\emptyset .
$$

Theorem 9.1 A meromorphic function of finite type is a rational function.

Proof. Let $\phi$ be a meromorphic function of finite type. Then there exists a rational function $h$ such that $\mathcal{L}_{\mathbb{R}}^{-}(h \otimes \phi)=\emptyset$. This means that every weight of facets is positive. Then from Corollary $9.3, h \otimes \phi$ is a rational function.

Remark This theorem shows that (after a suitable extension of the definition of a meromorphic function), a meromorphic function defined over a tropical projective space (in the sense of Kajiwara [4], Mikhalkin-Zhakov $[7])$ is a rational function since a tropical projective space is compact.

\section{Hilbert's nullstellensatz on hypersurfaces}

Hilbert's nullstellensatz in the following form is now easy to prove.

Theorem 10.1 Let $f, g \in \operatorname{Poly}\left(\mathbb{T}^{n}\right)$ be polynomial functions. Then if $V(f) \supset V(g)$ holds, then there exists a natural number $k \in \mathbb{Z}_{>0}$ and $a$ polynomial function $h \in \operatorname{Poly}\left(\mathbb{T}^{n}\right)$ such that

$$
f^{k}=g h .
$$

Proof. Since the statement is obvious if $f$ is a constant, we may assume $f$ to be nonconstant.

Note that the number of facets is finite. Thus take $k$ large enough so that every weight of facets of $f^{k} / g$ is positive.

Then from Corollary $9.3, h:=f^{k} / g$ is an element of Poly $\left(\mathbb{T}^{n}\right)$ and we have $f^{k}=g h$.

Remark The inverse also holds from Remark 7 and Remark 7.

Corollary 10.1 Let $F, G \in \mathbb{T}\left[x_{1}, \ldots, x_{n}\right]$ be polynomials satisfying $V(F) \supset V(G)$. Then there exists a natural number $k$ and a polynomial 
$H$ such that

$$
F^{k} \sim G \otimes H
$$

Theorem 10.2 Let $f, g \in \operatorname{Poly}\left[\mathbb{T}^{n}\right]$ be nonconstant functions satisfying the followings:

- $V(f)=V(g)$,

- every weight of a facet of $f$ equals the weight of the same facet of $g$.

Then $f=g$ holds up to constant.

Proof. Since $f / g$ has no facets, it is constant.

Corollary 10.2 Let $f \in$ Poly $[\mathbb{T}]$ be nonzero and put $V(f)=\left\{p_{1}, \ldots, p_{m}\right\}$, with the weight of each $p_{i}$ being $w_{i}$. Then

$$
f=\left(x \oplus p_{1}\right)^{w_{1}}\left(x \oplus p_{2}\right)^{w_{2}} \ldots\left(x \oplus p_{m}\right)^{w_{m}}
$$

holds up to constant.

Especially $\mathbb{T}$ is "algebraically closed".

Remark Because the irreducible decomposition of an element of Poly $\left(\mathbb{T}^{n}\right)$ is in general not unique, $V(f)=V(g)$ does not imply $f=g$ up to constant for two reduced polynomials $f$ and $g$. For instance,

$$
\begin{aligned}
& f=(x \oplus y \oplus 0)\left(x^{15} y^{21} \oplus y^{16} \oplus x^{8}\right) \\
& g=\left(x y^{3} \oplus y^{4} \oplus 0\right)\left(x^{3} y^{3} \oplus x^{2} \oplus y^{2} \oplus x\right)
\end{aligned}
$$

are reduced polynomials satisfying $V(f)=V(g)$, but are different.

On the other hand, we have $f^{4}=g h$ and $g^{8}=f h^{\prime}$ for the following $h$ and $h^{\prime}$ :

$$
\begin{aligned}
h & =\left(x^{3} y^{3} \oplus x^{2} \oplus y^{2} \oplus x\right)^{3}\left(x^{6} y^{6} \oplus x^{3} y^{9} \oplus y^{8} \oplus x^{4}\right)\left(x^{15} y^{21} \oplus y^{16} \oplus x^{8}\right)^{3}, \\
h^{\prime} & =(x \oplus y \oplus 0)^{7}\left(x^{3} y^{9} \oplus y^{8} \oplus 0\right)^{3} .
\end{aligned}
$$

Acknowledgments Special thanks are due to Professor Masanori Kobayashi for his warm and continuous encouragement in this work. The author thanks Professor Takeshi Kajiwara for the fruitful suggestions. The 
author also thanks the referee for careful reading for our manuscript and for giving useful comments.

\title{
References
}

[1] Gathmann A., Tropical algebraic geometry. Jahresbericht der DMV 108(1) (2006), 3-32.

[ 2 ] Gelfand I. M., Kapranov M.M. and Zelevinsky A.V., Discriminants, Resultants and Multidimensional Determinants (Birkhäuser, 1994).

[ 3 ] Hebisch U. and Weinert H. J., Semirings-Algebraic Theory and Applications in Computer Science (World Scientific, 1993).

[4] Kajiwara T., Tropical toric varieties, preprint (2006).

[ 5 ] Kapranov M. M., Amoebas over non-Archimedian fields, preprint (2000).

[6] Kirillov A. and Maeno T., Wonderful amoebas (Japanese). Sugaku 58 (2006) 151-164, translation in Sugaku Expositions.

[ 7 ] Mikhalkin G. and Zhakov I., Tropical curves, their Jacobians and Theta functions, preprint (math.AG/0612267).

[ 8 ] Mikhalkin G., Decomposition into pairs-of-pants for complex algebraic hypersurfaces. Topology 43(5) (2004), 1035-1065.

[ 9 ] Mikhalkin G., Amoebas of algebraic varieties and tropical geometry, in Different Faces of Geometry, ed. S. Donaldson et al. (Kluwer/Plenum, 2004), 257-300.

[10] Mikhalkin G., Enumerative tropical algebraic geometry in $\mathbb{R}^{2}$, J. Amer. Math. Soc. 18 (2005), 313-377.

[11] Oda T., Convex Bodies and Algebraic Geometry-An Introduction to the Theory of Toric Varieties (Springer-Verlag, 1988).

[12] Richter-Gebert J., Sturmfels B. and Theobald T., First steps in tropical geometry, in Idempotent Mathematics and Mathematical Physics, Proceedings Vienna 2003, ed. G. Litvinov and V. Maslov (American Mathematical Society), Contemp. Math. 377 (2005), 289-317.

[13] Rockafeller R. T., Convex Analysis (Princeton University Press, 1970).

[14] Rullgård H., Polynomial amoebas and convexity, preprint.

[15] Shustin E. and Izhakian Z., A tropical nullstellensatz, preprint (math.AC/ 0508413).

\author{
Department of Mathematics \\ Tokyo Metropolitan University \\ Tokyo 192-0397, Japan \\ E-mail: odagiri-shinsuke@ed.tmu.ac.jp
}

